\title{
Implementing Alternative
}

Pricing Policies in Economic Equilibrium Models Using

the Extended Mathematical Programming Framework

Bertrand Rioux, Abdullah Al Jarboua, Frederic Murphy, Axel Pierru 


\section{About KAPSARC}

The King Abdullah Petroleum Studies and Research Center (KAPSARC) is a non-profit global institution dedicated to independent research into energy economics, policy, technology and the environment across all types of energy. KAPSARC's mandate is to advance the understanding of energy challenges and opportunities facing the world today and tomorrow, through unbiased, independent, and high-caliber research for the benefit of society. KAPSARC is located in Riyadh, Saudi Arabia.

This publication is also available in Arabic.

\section{Legal Notice}

(C) Copyright 2020 King Abdullah Petroleum Studies and Research Center ("KAPSARC"). This Document (and any information, data or materials contained therein) (the "Document") shall not be used without the proper attribution to KAPSARC. The Document shall not be reproduced, in whole or in part, without the written permission of KAPSARC. KAPSARC makes no warranty, representation or undertaking whether expressed or implied, nor does it assume any legal liability, whether direct or indirect, or responsibility for the accuracy, completeness, or usefulness of any information that is contained in the Document. Nothing in the Document constitutes or shall be implied to constitute advice, recommendation or option. The views and opinions expressed in this publication are those of the authors and do not necessarily reflect the official views or position of KAPSARC. 


\section{Key Points}

tandard economic optimization models typically represent markets in perfect competition as linear programs (LPs). However, they do not accurately represent policies and regulations that exist in the real world. The extended mathematical programming (EMP) framework supports the implementation of a more general mixed complementarity problem (MCP), which can be used to:

Model non-competitive market structures and regulated pricing policies observed in the real world.

Automate the programming of optimality conditions that convert integrated LP models into MCPs, reducing the time and effort needed for model development and validation.

Support the parallel development of independent optimization problems, with varying economic and technical features, by providing a standard integration framework.

We provide a simple two-sector problem to explain how to introduce regulated pricing policies into integrated LP models. We also reference the open-source documentation of the KAPSARC Energy Model (KEM) available at https://kapsarc.github.io/kem/ to support larger-scale economic modeling. 


\section{Summary}

$\mathbf{S}$

tandard economic optimization models represent markets in perfect competition.

They are commonly used, though they may not accurately represent real-world policies and regulations. Equilibrium models built using a mixed complementarity problem (MCP) approach are capable of simulating more flexible pricing structures, including regulated prices. This approach can provide a more accurate representation of real-world economic systems. However, it is significantly more difficult to develop and requires more complex formulations, as well as advanced skill sets.

In this paper we explain how to introduce flexible pricing policies into economic optimization models using the extended mathematical programming (EMP) framework. The EMP framework developed by Ferris et al. (2009), available in the General Algebraic Modeling System (GAMS) (Brook 1988), can be used to automate the conversion of multiple linear programs (LPS) into an equilibrium framework. A collection of LPs representing different energy sectors, or agents in an economic system, can then be integrated using non-competitive pricing rules. This eliminates the need to manually program the optimality conditions of the MCP in GAMS, and reduces the time and effort required for model development and validation. It also supports the parallel development of independent optimization problems, with varying economic and technical features, by providing a simple and standard integration framework.

To illustrate how EMP works, we provide a simple two-sector example with several pricing rules, which collectively form a pricing policy library. They include the marginal cost (perfect competition), the administered (fixed) price, the average cost, and a price cap. We detail how to implement each case and reference applications to real-world markets. We also describe alternative market structures and their implementation in EMP, including budget constraints and more advanced option pricing contracts.

To give an example of how to incorporate EMP into larger scale energy system models, we reference the open-source documentation of the KAPSARC Energy Model (KEM). In addition, many popular economic modeling systems built in GAMS, such as The Integrated MARKAL-EFOM System (TIMES) developed under the Energy Technology Systems Analysis Program of the International Energy Agency (Loulou et al. 2016), could incorporate alternative pricing policies using the proposed framework. 


\section{Introduction}

O ver the last 40 years, computer models have become increasingly important in policymaking, as computer science and analytical software have advanced rapidly. Numerical economic modeling uses computers to simulate the impact of policies and human behavior on complex systems, such as integrated energy markets. Despite significant advances in modeling tools and techniques, it remains challenging to assess the efficiency and cost impacts of pricing policies and how they alter market outcomes.

Standard economic models generally assume competitive markets that maximize social welfare. However, this provides a limited representation of real-world markets that are subject to a variety of government interventions and other forces that distort market behavior. Such interference is especially prevalent in the energy sector, with significant impacts on both industry and households.

In recent decades mixed complementarity problem (MCP) models have supplanted linear programming models used in social science to model economic equilibria more generally. These types of models were initially used for economic problems in non-competitive markets, such as an oligopoly with non-integrable demand functions. This eliminated the need to iterate over linear programs to find an equilibrium because of the demand representation. Murphy et al. (2016) outline the justification for using an MCP approach to model regulated pricing policies.

The framework presented in this paper advances the transition to MCPs by reducing the effort needed to convert integrated optimization problems into an equilibrium model with non-competitive market features and price regulation.
The design logic is simple and begins with the direct link between optimization and equilibrium modeling, as presented by Enke (1951) and Samuelson (1953). The connection between the two can be seen by walking through the simplex algorithm (Dantzig 1968). Here, one views each activity in a linear program as an economic agent (or sector) with a positive activity level as long as the price of its output is greater than its marginal production cost. In mathematical terms, this is known as the complementarity slackness condition. In the simplex algorithm, an activity enters the solution when the profits are positive at the trial prices (trial duals) and increases until it changes the prices and the slackness condition holds. At the optimal solution, prices equal marginal costs and no activities have a positive profit (excluding classical economic rents).

In our approach, each sector is treated as a distinct economic agent and modeled as its own optimization problem that seeks to minimize costs or maximize profits. The sectors are then integrated by linking the aggregate demand with the supplies from the corresponding sector, referred to as cross-cutting activities, and selecting the desired pricing rule applied to each activity. The complementarity slackness conditions corresponding to the aggregate demand from each supplier can be designed differently from a standard optimization that sets the price equal to the marginal cost. In this paper we describe how to incorporate four basic pricing structures into multi-sector economic problems: the marginal cost (perfect competition), the administered (fixed) price, the average cost price, and a price cap. For a more detailed explanation of how MCP models can be used to measure the effects of price controls, see Murphy et al. (2019). 
We use the extended mathematical programming (EMP) framework to automate the construction of the MCP for each sector. The price linkages connecting the cross-sector activities are introduced as independent equilibrium conditions, representing the rules set by the market regulator.

The proposed framework for constructing the pricing library can be applied to a variety of real-world market structures. For example, in Saudi Arabia, industrial prices for crude oil and natural gas are fixed at levels well below their marginal supply value (e.g., the prices available on the international market), as modeled by Matar el al. (2015; 2017). In China's wholesale natural gas market, price caps and contractual obligations are enforced on pipeline deliveries at regulated prices, while prices for LNG contracts are unregulated. In the model developed by Rioux et al. (2018), the EMP framework was used to represent the profit maximizing incentive for suppliers not to sell pipeline gas at a price capped below the marginal supply cost.

EMP can also facilitate the construction of budget constraints applied to multi-sector models. In their analysis of China's wholesale electricity market, Rioux et al. (2017) create a multi-regional model of the coal and power supply network that incorporates a budget constraint to capture the impact of price caps on operation and investment decisions by generators. In this case, EMP helps construct a budget constraint that aggregates the operating costs of different power suppliers, including the value of coal purchased by power generators at competitive market prices, represented by a dual variable for the demand balance of coal.

The pricing library can also be used to simulate taxation or international trade tariff policies in network economics. Nagurney (1999) provides a thorough overview of network problems in partial and general equilibrium models, with application to ad-valorem taxes. Two examples include modeling carbon taxes in electric power networks (Nagurney et al. 2006) and coal taxes as a supply-side carbon emissions policy (Richter et al. 2018).

In the following section, we provide a brief history of the development of energy system models. We then formulate a simple two-sector optimization problem, consisting of a fuel provider and a power generator, and derive the optimality conditions that capture both fixed and competitive fuel pricing policies. This is followed by a description of how EMP can be used to automate the formulation of the optimality conditions derived for the two-sector problem. Next, we explain how to use the EMP framework to construct each pricing option. Finally, we develop a model integration framework that can support the selection of multiple pricing rules. 


\section{A Brief History of Economic Equilibrium Models}

$\mathbf{E}$ arly economic equilibrium problems were solved using linear and nonlinear programming because the maximum economic surplus is achieved when supply meets demand, with prices equal to marginal supply costs (Samuelson 1953; Enke 1951; Takayama and Judge 1964). The Brookhaven Energy System Optimization Model (BESOM) (Hoffman and Jorgenson 1977), a linear program, was designed to analyze the potential penetration of technologies in the United States. The other prominent energy model of that era was the Project Independence Evaluation System (PIES) (Hogan 1975).

BESOM uses marginal costs as prices because its intended application was long-run technology investments rather than immediate short-term regulatory concerns. However, PIES was designed to address the regulatory issues of the 1970s: gas and oil price controls, average-cost electricity pricing, technological and legal barriers to addressing environmental problems and fuel shortages, and end-use price controls on fuels. These conditions meant that fuel and energy prices were not equal to the marginal costs, and a linear programming solution could not estimate the consequences of policy changes. Although PIES employs linear programs to represent fuel supplies, the model was solved using a step-in algorithm that iterated between the demand representation under price regulation and the supply structure. In the iterations, prices based on marginal costs were adjusted to average or controlled prices to find an equilibrium in the regulated market (Greenberg and Murphy 1985; Murphy et al. 1981). A similar technique can be used to find an equilibrium in an oligopolistic market formulated as an optimization problem (Murphy et al. 1982).

Nash equilibrium models have grown in popularity following improvements in solution algorithms designed to solve MCPs. These have since replaced the iterative methods used to solve equilibrium problems via standard optimization. To contribute to the evolution of solution methods for economic equilibrium models, this paper focuses on how to build pricing policy models using an MCP approach, and automate their formulation in GMAS using EMP. 


\section{A Simple Two-Sector Problem}

W

e introduce a simple two-sector example to illustrate how the EMP framework can be used to construct the pricing options available in the pricing library. An upstream supplier minimizes the cost of producing fuel sold to a utility company at a fixed price, to transform it into electric power (or other products). The indices, variables and coefficients of the two sectors are summarized in Figure
1. We identify $f_{j}$ as the fuel produced from each supply step $j$ at marginal production cost $C_{j}$. The coefficient $H_{i}$ defines the heat rate of technology $i$ for the consumption of fuel used in the generation of electricity $x_{i}$. The variable $p$ is the fuel price paid by the utility. The optimization problems of the fuel supplier and power company are represented in equation blocks (1) and (2), respectively.

Figure 1. Variables and coefficients used in the two-sector optimization problem.

\begin{tabular}{lll} 
Variables & & \\
\hline$z_{\text {fiel }}$ & Objective value of the fuel supply sector & $\mathrm{z}_{\text {fuel }}$ \\
\hline$z_{\text {power }}$ & Objective value of the utility company & $\mathrm{z}_{\text {power }}$ \\
\hline$f_{j}$ & Fuel supply from step $j=\{1,2\}$ for supplies with different costs & $\mathrm{f}(\mathrm{j})$ \\
\hline$F$ & Total fuel consumed by the utility & $\mathrm{Fx}$ \\
\hline$x_{i}$ & $\begin{array}{l}\text { Capacity supplied by utility for technology } i=\{G T, C C\} G T=G a s \\
\text { Turbines, } C C=\text { Combined } C y c l e ~ G T\end{array}$ & $\mathrm{x}(\mathrm{i})$ \\
\hline$p$ & Price of fuel purchased by the utility company & $\mathrm{P}$ \\
\hline$\pi$ & Dual variable for the fuel demand constraint $(1.3)$ & $\mathrm{pi}$ \\
\hline Coefficients & & $\mathrm{A}$ \\
\hline$A$ & The fixed price of fuel paid by the utility company & $\mathrm{C}(\mathrm{j})$ \\
\hline$C_{j}$ & The marginal production cost of fuel from supply step $j$ & $\mathrm{H}(\mathrm{i})$ \\
\hline$H_{i}$ & $\begin{array}{l}\text { Heat rate for consumption of fuel by technology } i \text { owned by the } \\
\text { utility }\end{array}$ & $\mathrm{D}$ \\
\hline$D$ & Total demand for electricity & $\mathrm{S}(\mathrm{j})$ \\
\hline$S_{j}$ & Upper bound on the available fuel in supply step $j$ & \\
\hline & & \\
\hline
\end{tabular}




$$
\begin{aligned}
& \min _{f_{j} \geq 0} z_{\text {fuel }}\left(f_{j}\right)=\sum_{j} f_{j} C_{j} \text { s.t. } \\
& 0 \leq f_{j} \leq S_{j} \\
& \sum_{j} f_{j} \geq F \\
& \min _{x_{i} \geq 0} z_{\text {power }}\left(x_{i}\right)=F \cdot p \text { s.t. } \\
& D-\sum_{i} x_{i} \leq 0 \\
& F=\sum_{i} x_{i} H_{i} \\
& p=\left\{\begin{array}{l}
\pi \\
A
\end{array}\right.
\end{aligned}
$$

In (1) the fuel sector minimizes the total supply costs $z_{\text {fuel }}$, optimizing $f_{j}$ subject to different constraints. In equation (1.1) the coefficient $S_{j}$ sets the upper bound on the level of fuel extracted from each supply step $j$. The demand balance constraint (1.2) requires that fuel production exceeds the exogenous consumption of fuel $F$ by the utility. From the perspective of the fuel supplier, $F$ is an exogenous coefficient defined as the aggregate of the utility's power production multiplied by the heat rate in equation (2.2). The fuel demand constraint is matched with a dual variable, also referred to as the marginal value, in this case of producing fuel.

In (2) the utility company minimizes the cost of purchasing fuel, optimizing the generation of electricity $x_{i}$ to satisfy fixed demand $D$ in (2.1). Equation (3) sets the price paid for fuel as either the marginal supply cost or a fixed price coefficient $A$. This is an equilibrium condition separate from the optimization problems, and represents actions taken by the market regulator. Other functional forms, such as average cost pricing or a maximum price, are explored later.

In order to solve (1) and (2) simultaneously, while satisfying the equilibrium conditions (2.2) and (3) that link them, we derive the optimality condition of the linear complementarity problem (LCP) for each. This is done using the Karush-Kuhn-Tucker (KKT) approach in accordance with duality theory, by assigning an orthogonal dual variable to the constraints of the original optimization (primal) problems, such as $\pi$ in (1.2). When the constraints are binding the dual variables can be positive.

The objective function, primal constraints and dual variables are connected through a Lagrangian function. The definition of the Lagrangian and derivation of each LCP is provided in Appendix 1. An equilibrium solution is found by solving for the Lagrangian's stationarity, or optimality, conditions with respect to the primal variables. This expresses the dual variables in terms of the cost coefficients of the objective function (i.e., unit costs or prices), and coefficients from each constraint, producing a balanced number of variables and equations. To complete the exercise, we combine each LCP and the price linkage (3), producing the MCP in (4). When the price linkage (4.6) is set to the marginal cost $\pi$, the MCP is equivalent to the LCP of the combined optimization problems of (1) and (2).

The following dual variables are assigned to each primal constraint: $\mu_{j}$ for (1.1), $\pi$ for (1.2), and $\lambda$ for (2.1). We link the identity in (3) with the price itself; as an equality, its dual is not constrained and can take on any value. We substitute identity (2.2) for the 
utility's fuel consumption variable $F$, simplifying the system to five variables and equation pairs and the identity (4.6). Although (2) contains a bilinear term when $p=\pi$, this is a standard Lagrangian used to construct the LCP, with primal variables multiplied by dual variables.

(4)

$\begin{array}{ll}C_{j}+\mu_{j}-\pi \leq 0 & \perp f_{j} \geq 0 \\ H_{i} p-\lambda \leq 0 & \perp x_{i} \geq 0 \\ f_{j}-S_{j} \leq 0 & \perp \mu_{j} \geq 0 \\ \sum_{j} f_{j} \geq \sum_{i} x_{i} H_{i}=F & \perp \pi \geq 0 \\ D-\sum_{i} x_{i} \leq 0 & \perp \lambda \geq 0 \\ p=\left\{\begin{array}{l}\pi \\ A\end{array}\right. & \perp p\end{array}$

Manually coding the optimality conditions can take a significant time for a large set of optimization problems consisting of multiple sectors with many variables and constraints. Validating the optimality conditions adds further complexity. In addition, transforming each optimization problem into the corresponding equilibrium conditions can mask the economic inputs and rationale that they are built on. For example, what are the exact terms included in the original objective functions? And are the problems cost minimizing, profit maximizing or a combination of both?

The manual construction of the MCP also adds additional work when modifying the model, because both the primal and dual constraints must be updated and verified. Automating the derivation of the MCP offers a more efficient approach to managing changes, while also preserving the economic intuition applied to each sector in an integrated model. 


\section{Formulating the Optimality Conditions in EMP}

$\mathbf{T}$

The EMP framework was developed as a general approach to constructing mathematical equilibrium problems within GAMS. The components of different optimization problems are passed through a model compiler known as JAMS (Ferris et al. 2009) that reformulates the independent primal problems as a single MCP. The primal problem is reconstructed using scalar elements, rather than the multi-dimensional variables generally used in GAMS. New scalar dual variables are assigned to each primal constraint to construct the Lagrangian and formulate the optimality conditions. JAMS will also generate a model dictionary to connect the scalar elements with the symbolic variables and equations of the original optimization problem.

We use EMP to demonstrate how to generate the MCP presented in equation block (4). First, we formulate the optimization problems (1) and (2) in GAMS (see code provided in Appendix 2). We generate the optimality conditions corresponding to competitive pricing (other pricing structures, and the corresponding equations and code, are described later.)

For reference, the GAMS notation used for each variable and coefficient is defined on the right-hand side of Figure 1. In Figure 2 we show the lines of GAMS code used to construct the equilibrium model with EMP. In the GAMS code we use the symbols EQ1_1, EQ1_2, EQ2_1, EQ3 and EQ4 to represent equations (1.1), (1.2), (2.1), (2.2) and (3), respectively. The objective functions for problems (1) and (2) are expressed as EQ_z_fuel and EQ_z_power.

First, a new file called emp.info is created (line 1). Text is appended using the PUT statement, and the file is closed using PUTCLOSE (line 11). Line 2 defines the type of model we want EMP to generate, in this case an equilibrium problem (see Ferris et al. [2009] for more information).

Figure 2. The EMP configuration file.

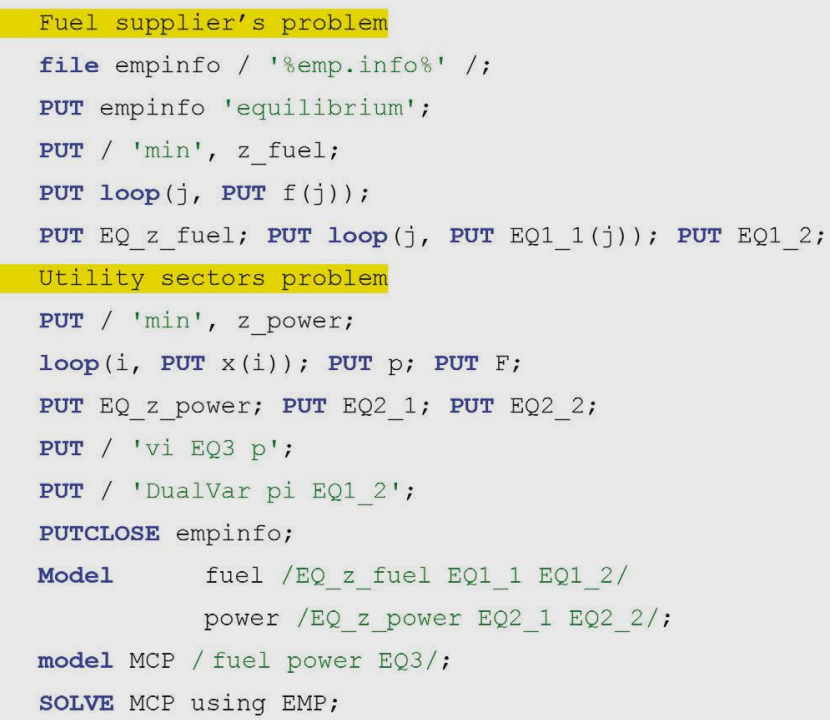


Next we list elements of the fuel supplier (lines 3, 4 and 5) and the utility (lines 6, 7, and 8). This must be done in the following sequence: declare the direction of the optimization (max or $\min$ ) applied to the objective value, z_fuel or z_power; compile a list of all the variables, and finally compile a list of all the constraints belonging to the sector. Multidimensional symbols are entered within a loop statement to add each set element of $i$ and $j$ to the file.

After defining each sector's problem, EMP allows the user to introduce additional equilibrium conditions, referred to as 'variational inequalities' that stand as separate agents. We introduce equilibrium condition (3) for variable $p$, using the symbol vi in EMP (line 9). EMP also allows for optional dual-variable and dual-equation statements. These are used to help construct the pricing options. We define pi as the dual variable in the fuel demand balance (1.2) (line 10) used to configure the competitive market option.
Finally, we combine the fuel and power sectors into a single model labeled MCP (lines 12 to 14). On the last line (15) the model solve statement tells EMP to pass the emp.info to the JAMS compiler that outputs the MCP represented in equation block (4). Figure 3 provides the dictionary generated by JAMS that matches the scalar primal equations and variables, as generated by the EMP statement in Figure (2), to the original primal problems, as well as the additional dual variables and equations used to build the MCP. All primal constraints start with $e$, primal variables and duals declared in emp.info (pi) with $x$, new dual variables with $u$, and dual constraints with $d L \_d x$. Each dual variable and constraint carries the same sequential number as the corresponding primal. Reformulated equations and variables refer to elements that are not included in the final MCP model, such as the objective functions. The final MCP output by EMP is shown in Figure $4 .{ }^{1}$

Figure 3. Dictionary of scalar elements generated by JAMS.

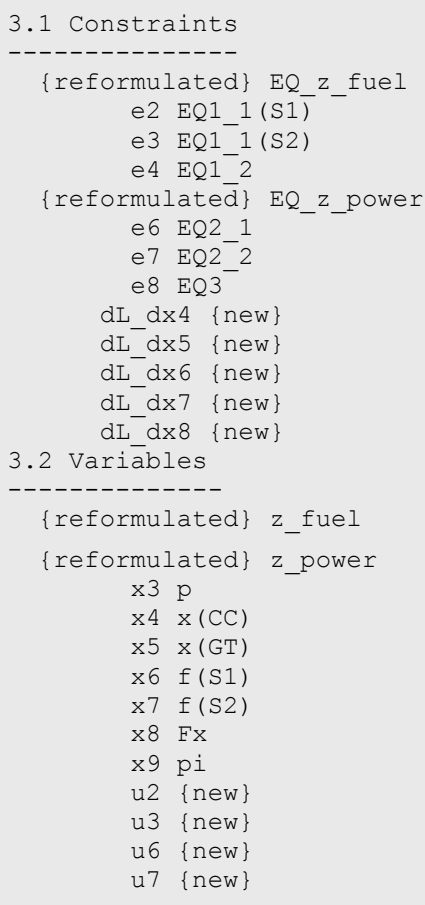


In practice, constructing the emp.info file for a large model with many sectors can be a challenge of its own. However, this can be resolved by using a standardized syntax and additional programming tools to identify and extract the components of each LCP and automate the construction of the emp.info file. For example, specific prefixes identify the components of each sector and post fixes identify independent agents within each sector (e.g., countries). When introducing EMP into the KAPSARC Energy Model (KEM), a python script is used to help build the emp.info based on our standard naming convention for variables and equations. A complete description of how the EMP statement is constructed can be found online (KEM Manual 2019).

Figure 4. Scalar model generated by JAMS.

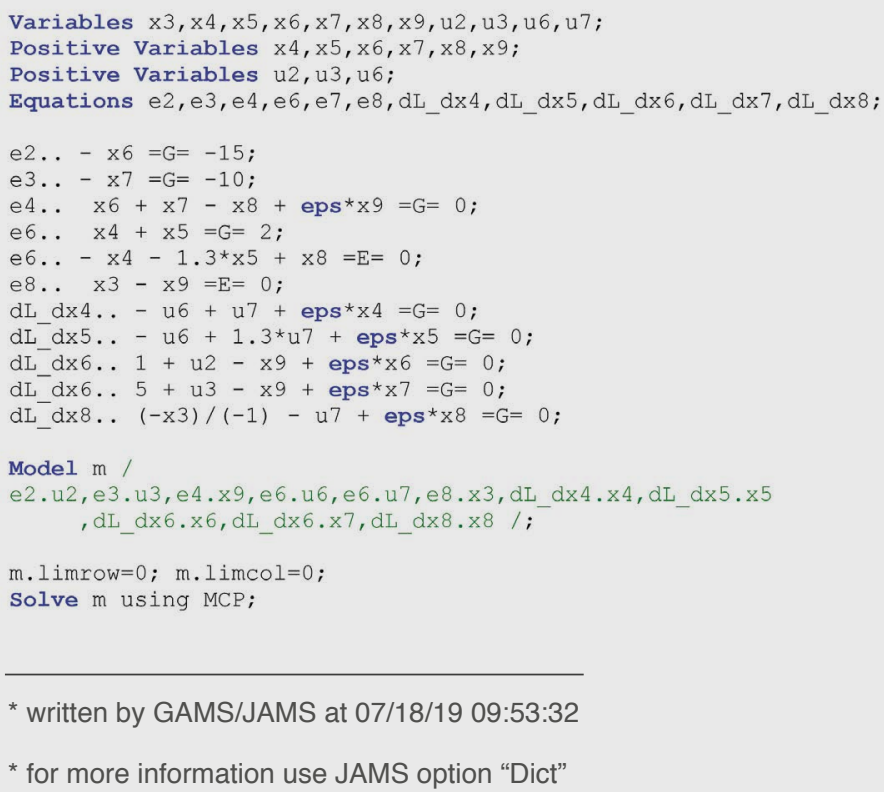

(1). Terms containing eps, a GAMS reserved symbol for a small number less than model tolerance, are used to identify variables declared in the EMP as the complements to the corresponding equation. 


\section{Extending the Pricing Library}

$\mathrm{n}$ this section, we describe how to design additional pricing policy options using EMP. We

describe four pricing rule categories:

Marginal cost (perfect competition)

Administered (fixed) pricing

Average cost pricing

\section{Price cap}

Before going further, it is important to address market clearing issues that arise when prices do not reflect marginal costs. When supplies are limited, and the price paid by consumers is regulated below the marginal cost, supplies may be insufficient to meet the quantities that would be in demand at that price. A simple solution is to relax the supply constraints. For example, a government could import a domestically scarce resource and pay a subsidy to consumers. However, this may not be a valid solution for political, economic or other reasons. A second approach is to allocate a quota of the scarce resource to each sector, a common practice in regulated markets. This is represented in the model as a quantity constraint, as introduced in equations (5.2) and (5.4) below for the price cap policy.

Both the competitive (marginal) and administered (fixed) price options have been defined in equation (3) as $\pi$ and $A$, respectively. The average-cost pricing rule provides more flexibility by defining the price using an aggregate measure of the system costs, so that the price becomes a function of the supplier's activities, for example $p=\sum_{j}\left(C_{j} f_{j}\right) / \sum_{j} f_{j^{*}}$

In the last option, the price cap $\bar{p}$ sets the maximum fuel price paid by the utility, $p=\min (\pi, \bar{p})$. When the marginal value falls below the cap, the utility pays the standard competitive price. Since this function is discontinuous, it is reformulated as a set of complementary slackness conditions in (5.1), (5.2) and (5.3) using the components listed in Figure 4.

Figure 5. Variables and parameters for the price cap policy.

\begin{tabular}{|c|c|c|}
\hline $\bar{p}$ & Price cap in the hybrid market & p_cap \\
\hline$Q$ & Quota levels allocated by the regulator & Quota \\
\hline \multicolumn{3}{|c|}{ Variables } \\
\hline$\sigma$ & Non-negative difference $\pi$ - $\bar{p}$ for $\pi>\bar{p}$ & sigma \\
\hline$q$ & Enforcement of quotas by the regulator, $Q$ & $\mathrm{q}$ \\
\hline$r$ & $\begin{array}{l}\text { Slack variable relaxes the quota constraint }(5.4) \text { when the price cap is not } \\
\text { binding }\end{array}$ & $\mathrm{r}$ \\
\hline
\end{tabular}




$\begin{array}{ll}\sigma \geq \pi-\bar{p} & \perp q \geq 0 \\ q \leq Q & \perp \sigma \geq 0 \\ \sigma \geq 0 & \perp r \geq 0 \\ \sum_{i} x_{i} H_{i} \leq q+r & \end{array}$

To construct the price cap policy, we introduce two quantity slack variables. First we define the volume of fuel quotas enforced by the regulator when the price cap is binding as the variable $q$. Second we introduce $r$ to relax the amount of fuel that can be consumed by the utility if the price cap is not binding and the utility is not bound to the quota.

Equation (5.1) is the slackness condition for the enforcement of quotas $q$ by the regulator. The non-negative slack variable $\sigma$ measures the difference between the cap and the marginal value when the cap is binding $(\pi \geq \bar{p})$. Constraint (5.2) signals the regulator to enforce supply quotas $(q=Q)$, where $Q$ is the predefined allocation. If not binding $(\pi<\bar{p})$, quotas are not enforced $(q=0)$, and $r$ in (5.3) is used to relax the quantity available for consumption. In this case the marginal supply cost sets the market price and determines the efficient level of consumption by the utility. The variables $q$ and $r$ are combined in equation (5.4) to set the upper bound on the utility's fuel consumption. Note that when
(5) applying a fixed or average cost price, one can use (5.4) as the quantity constraint by replacing the variables on the left-hand side with the fixed quota $Q$ assigned to the sector.

$p=\pi-\sigma$

To include the price cap option, we add variables $q$ and $r$, and conditions (5.1) - (5.4) to the emp.info file under the utility sector's problem. Equation (6) is introduced as an additional identity in the original equilibrium condition (3), so that the price never exceeds the cap. We assign $\sigma$ as the dual variable on equation (5.2). Constraints (5.1) and (5.3) are declared as dual equations for $q$ and $r$, respectively, that are imposed rather than derived from the Lagrangian. The optimization problems for the two-sector model with all four pricing options are provided in Appendix 2, followed by the complete EMP statement used to construct and solve the MCP.

The concept of a price cap can also be replaced by a minimum price. In this case $\bar{p}$ is redefined as a lower price bound swapping its position with $\pi$ in (5.1). From the perspective of the buyer, the slack variable $q$ in (5.2) can be redefined as a lower bound, representing the buyer's requirement to purchase the pre-determined obligation $Q$. 


\section{Reliability Options}

M odels with more complex pricing mechanisms, such as reliability options used to provide long-term supply adequacy in electricity markets (Bidwell 2005), can also be constructed using EMP. Options are financial contracts that give the holder the right, without obligation, to buy or sell a quantity of an underlying asset at a specified price either before or on a future expiration date. In this section we construct an equilibrium problem in which competing power generators sell rights to portions of their capacities to the wholesale market as reliability options. These contracts are generally long-dated, possibly for a year or more from the sale date. They benefit generators by providing cash up front to manage investment and operating costs, while allowing market operators to hedge against expected price spikes during periods of high demand.

Reliability options have been used in the Iberian electricity market (Garcia et al. 2017). Lynch and Devine (2017) propose a model of reliability options using a stochastic MCP model to investigate their impact on investment and retirement decisions by power generation companies. We design a similar stochastic MCP and show how to implement it in EMP in Appendix 3.

Figure 6. Variables and parameters used for the reliability options model.

\section{Coefficients}

\begin{tabular}{ll}
$C_{i j k}$ & Marginal cost of generation technology $i$ for generator $j$ in scenario $k$ \\
\hline$E_{i j}$ & Existing capacity owned by generator $j$ in technology $i$ \\
\hline$\hat{p}$ & The strike price of the options contract \\
\hline$\rho_{k}$ & Probability of each scenario $k$ \\
\hline Variables & The price of the option contract for capacity committed to load segment $l$ \\
\hline$o_{l}$ & Capacity exercised by the buyer for all options contracts in scenario $k$ and segment $l$ \\
\hline$q_{k l}$ & Wholesale market price for energy in scenario $k$ and load segment $l$ \\
\hline$p_{k l}$ & Non-negative difference between $p_{k l}$ and $\bar{p}$ \\
\hline$s_{k l}^{+}$ & Capacity sold into the options contract in advance by generator $j$ load segment $l$ \\
\hline$r_{j l}$ & Energy supplied by technology $i$ and generator $\mathrm{j}$ in scenario k under load segment $l$ \\
\hline$x_{i j k l}$ & \\
\hline
\end{tabular}


We introduce a set of generation companies $j$ that compete to sell energy into a wholesale energy market supplied by technologies $i$ for different load segments $l$ and scenarios $k$. The scenarios represent stochastic conditions in the wholesale market, such as variability in demand or the marginal cost of production $C_{i j k}$, with probability distribution $\pi_{k}$.

Each power company owns the existing capacity $E_{i j}$ as the upper bound on the variable $x_{i j k l}$ for energy supplied to the wholesale market. This capacity can also be sold into reliability options, variable $r_{j l}$, at options price $o_{l}$ as determined by the model. The reliability options commit the capacity to the predefined exercise price $\hat{p}$ ahead of the wholesale market. Finally, the variable $q_{k l}$ represents the pool of contracts exercised by wholesale buyers.

We define the components of the wholesale market with reliability options in (6), (7) and (8) below. Figure 6 lists all the coefficients and variables used.

$p_{k l}=a_{k l}-b_{k l} \sum_{i j} x_{i j k l}$

In equation (6) the wholesale energy price is defined as an inverse demand function with respect to the total energy supplied $\sum_{i j} x_{i j k l}$. The coefficients $a_{k l}$ and $b_{k l}$ are the slope and intercept of the inverse demand curve, respectively. They are calibrated to observed conditions in the wholesale energy market, such as the expected price elasticity of demand in a given load segment.

\section{The reliability options}

$\begin{array}{ll}s_{k l}^{+} \geq p_{k l}-\hat{p} & \perp q_{k l} \geq 0 \\ \sum_{j} r_{j l} \geq q_{k l} & \perp s_{k l}^{+} \geq 0 \\ o_{l}=\sum_{k} s_{k l}^{+} \rho_{k} & \end{array}$

We construct a set of equations in (7) describing the reliability options sold to wholesale market operators. We introduce the slack variable, $s_{k l}^{+}$, as the non-negative difference between the energy price and the strike price, $\max \left(0, p_{k l}-\hat{p}\right)$. We linearize this expression using the set of complementarity slackness conditions in (7.1) and (7.2). Together they state that when the energy price is at least the exercise price $\left(s_{k l}^{+} \geq 0\right)$, the capacity exercised by the wholesale buyers $q_{k l}$ is equal to the sum of the capacity rights $\left(\sum_{j} r_{j l}\right)$ sold into all options by the generators, equation (7.2). When the energy price is less than the cap the capacity exercised is 0 .

In (7.3) the price of the reliability options $o_{l}$ is calculated as the expected value of the slack variables $s_{k l}^{+}$. This is the expected market value that the generator gives up when selling capacity into reliability options ahead of the wholesale energy market.

Notice that the use of the strike price $\hat{p}$ is similar to the price cap described in (5). However, here the market determines the value and sets the pool of capacity $\sum_{j} r_{j l}$ committed by the generators, rather than being set by a regulator.

We introduce the generator's problem (8), with the objective to maximize revenues $z_{j}$ from selling reliability options plus the expected profit from supplying the energy market. The last term represents the wholesale market value reimbursed by the generator $\left(s_{k l}^{+}>0\right)$ for all reliability options $r_{j l}$ exercised by the buyer.

\section{The generator's problem}

$\max z_{j}\left(x_{i j k}, u_{j l}, v_{j k l}\right)=\sum_{l} r_{j l} \cdot o_{l}+\sum_{i k l}\left(p_{k l}-C_{i j k}\right) x_{j k l} \rho_{k}-\sum_{k l} s_{k l}^{+} r_{j l} \rho_{k}$ s.t.
$x_{i j k} \geq 0$
$\sum_{i j^{\prime}} x_{i j^{\prime} k l} \geq q_{k l}$
$E_{i j} \geq x_{i j k l}$
$\sum_{i} E_{i j} \geq r_{j l}$


Equation (8.1) is a shared constraint of all the generators, where $j^{\prime} \equiv j$, enforcing the capacity contracts exercised by the buyer as a lower bound on the total energy supplied by all generators. Equation (8.2) is the capacity constraint on the power generated from each technology in a given scenario and load segment. In (8.3) the capacity sold by the generator into a contract for a given load segment, $r_{j l}$, must not exceed the total available capacity.

Next, we derive the equilibrium problem for reliability options within a wholesale electricity market. First the optimality conditions are derived for the generator's problem (8) and combined with the reliability option constraints in (6) and (7). We use EMP to automate the construction of the corresponding MCP of competing generators, solvable as a numerical problem with real-world data. A complete derivation of the MCP and how to implement it in GAMS and EMP is provided in Appendix 3.

The generator's problem described above can also be formulated as a Cournot oligopoly, a prevailing model of imperfect competition (e.g., Frederic et al. 1982; Khalfallah 2011; Rioux et al. 2018). In such a market, suppliers strategically withhold capacity to increase prices and marginal revenues. Since the reliability options commit generation capacity in advance of the wholesale market, they can help mitigate the impact of such manipulation. Non-competitive behavior goes beyond the pricing policy design focus of this paper. However, we demonstrate how to use EMP to derive the Cournot oligopoly within our reliability options model in Appendix 3. 


\section{Conclusion and Future Work}

W

e have presented a new framework to incorporate a library of pricing policy options into economic equilibrium models. A simple example was presented for the sale of fuel to a power utility with different fuel pricing structures, demonstrating how the EMP framework by Ferris et al. (2009) can automate the formulation of an equilibrium problem. We showed how to build a pricing library with perfectly competitive, administered, average cost, and capped pricing policies. Real-world applications of such pricing libraries were discussed, and we demonstrated how to construct a problem involving reliability options used in liberalized electricity markets. EMP can be applied to a variety of economic pricing problems, although it is currently only available in GAMS.

Applying the EMP framework requires a strong grasp of how MCP models are formulated but reduces the amount of coding needed to build the complete optimality, or KKT, conditions. We recognize that learning how to use the EMP framework for this class of problems comes with its own learning curves and challenges. To help overcome these issues, we illustrated the basic approach needed to implement largescale numerical models of real economies with different pricing structures. The GAMS code references provided in appendices 2 and 3 are available from the KEM manual (KAPSARC 2018) at https://kapsarc.github.io/kem/ Additional online documentation for KEM offers design recommendations and an open source code based on the EMP framework. This code is freely available for others to work on, extending the capabilities and real-world applications of KEM, or to incorporate new pricing paradigms into existing integrated optimization models built in GAMS, such as the TIMES modeling system. 


\section{References}

Bidwell, Miles. 2005. "Reliability Options: A MarketOriented Approach to Long-term Adequacy." The Electricity Journal 18(5): 11-25. https://doi.org/10.1016/j. tej.2005.03.010

Brook, Anthony, David Kendrick, and Alexander Meeraus. 1988. "GAMS, a User's Guide." SIGNUM Newsletter. 23(3-4): 10-11.

Dantzig, George B. 1968. "Large-Scale Linear Programming," in George B. Dantzig and Arthur F. Veinott, Jr. (eds.), Mathematics of the Decision Sciences, the American Mathematical Society Summer Seminar, Providence, R.I., 77-92.

Dirkse, Steven. 2011. "SPE Model from Harker Oligopoly Version.” June. https://www.gams.com/latest/ emplib_ml/libhtml/emplib_hark-oligop.html.

Enke, Stephen. 1951. "Equilibrium among Spatially Separated Markets: Solution by Electric Analogue." Econometrica 19: 40-46. https://doi.org/10.2307/1907907

Ferris, Michael C., Steven P. Dirkse, Jan-Hendrick Jagla, and Alexander Meeraus. 2009. "An Extended Mathematical Programming Framework." Computers \& Chemical Engineering 33(12): 1973-1982. https://doi.org/ https://doi.org/10.1016/j.compchemeng.2009.06.013.

Garcia, Augustin, Maria Teresa Garcia-Alvarez, and Blanca Moreno. 2017. "Iberian Electricity Sector: A Transition Towards a More Liberalized and Sustainable Market." Global Development and Environment Institute. Working paper 17-01. Tufts University.

Greenberg, Harvey J., and Frederic H. Murphy. 1985. "Computing Market Equilibria with Price Regulations Using Mathematical Programming." Operations Research 33(5): 935-954. https://doi.org/10.1287/opre.33.5.935.
Hoffman, Kenneth C., and Dale Jorgenson. 1977. "Economic and Technological Models for Evaluation of Energy Policy." The Bell Journal of Economics. 8(2): 444-466.

Hogan, William W. 1975. "Energy Policy Models for Project Independence." Computers \& Operations Research 2(3-4):251-271. https://doi. org/10.1016/0305-0548(75)90008-8

KAPSARC. 2018. "KAPSARC Energy Model Manual" (KEM). Accessed October 21, 2019. https://kapsarc. github.io/kem.

Khalfallah, Mohammed H. 2011. "A Game Theoretic Model for Generation Capacity Adequacy: Comparison between Investment Incentive Mechanisms in Electricity Markets." The Energy Journal 32(4): 117-157. http://dx.doi. org/10.5547/ISSN0195-6574-EJVol32-No4-7.

Loulou, Richard, Gary Goldstein, Amit Kanudia, Antti Lettila, and Uwe Remme. 2016. "Documentation for the TIMES Model: Part I." IEA Energy Technology Systems Analysis Program. https://iea-etsap.org/index.php/ documentation

Lynch, Murieann A., and Mel T. Devine. 2017. "Investment vs. Refurbishment: Examining Capacity Payment." The Energy Journal 38(2): 27-51. https://doi. org/10.5547/01956574.38.2.mlyn.

Matar, Walid, Frederic Murphy, Axel Pierru, and Bertrand Rioux. 2015. "Lowering Saudi Arabia's fuel consumption and energy system costs without increasing end consumer prices." Energy Economics, 49: 558-569. https://doi.org/10.1016/j.eneco.2015.03.019

- - - 2017. "Efficient industrial energy use: The first step in transitioning Saudi Arabia's energy mix." Energy Policy 105: 80-92. http://doi.org/10.1016/j. enpol.2017.02.029 
Murphy, Frederic H., Reginal C. Sanders, Susan H. Shaw, and Richard L. Thrasher. 1981. "Modeling Natural Gas Regulatory Proposals Using the Project Independence Evaluation System." Operations Research 29: 876-902. https://doi.org/10.1287/opre.29.5.876.

Murphy, Frederic H., Hanif D. Sherali, and Allen L. Soyster. 1982. "A Mathematical Programming Approach for Determining Oligopolistic Market Equilibrium." Mathematical Programming 24: 92-106. https://doi. org/10.1007/BF01585096.

Murphy, Frederic, Axel Pierru, and Yves Smeers. 2016. "A Tutorial on Building Policy Models as MixedComplementarity Problems." Interfaces 46(6): 465-81. https://doi.org/10.1287/inte.2016.0842

Murphy, Frederic, Axel Pierru, and Yves Smeers. 2019. "Measuring the Effects of Price Controls Using Mixed Complementarity Models." European Journal of Operational Research 725(1): 666-676. https://doi. org/10.1016/j.ejor.2018.11.051

Nagurney, Anna. 1999. "Network Economics: A Variational Inequality Approach." Book 1 of Advances in Computational Economics Vol. 10. Dordrecht: Springer.
Richter, Brian, Roman Mendelevitch, and Frank Jotzo. 2018. "Coal taxes as a supply-side climate policy: a rationale for major exporters?" Climatic Change 150:4356. https://doi.org/10.1007/s10584-018-2163-9

Rioux, Berrtand, Philipp Galkin, Frederic Murphy, and Axel Pierru. 2017. "How do Price Caps in China's Electricity Sector Impact the Economics of Coal, Power and Wind? Potential Gains from Reforms." The Energy Journa/ 38. https://doi.org/10.5547/01956574.38.SI1.brio

Rioux, Bertrand, Philipp Galkin, Frederic Murphy, Axel Pierru, Artem Malov, Felipe Feijoo Palacios, Yan Li, and Kang Wu. 2019. "The Economic Impact of Price Controls on China's Natural Gas Supply Chain”. Energy Economics 80: 394-410. https://doi.org/10.1016/j. eneco.2018.12.026

Samuelson, Paul A. 1953. "Prices of Factors and Goods in General Equilibrium." The Review of Economic Studies 21(1): 1-20. https://doi.org/10.2307/2296256

Takayama, Takashi, and George G. Judge. 1964. "Equilibrium among Spatially Separated Markets: A Reformulation." Econometrica 32: 510-524. https://doi. org/10.2307/1910175

Nagurney, Anna, Zuguang Liu, and Trisha Woolley. 2006. "Optimal Endogenous Carbon Taxes for Electric Power Supply Chains with Power Plants." Mathematical and Computer Modelling 44(8): 899-916. DOI: https://doi. org/10.1016/j.mcm.2006.02.018 
The mixed complementarity problem (MCP) that combines problems (1) and (2) and the price identity (3) is derived using duality theory. First we write the Lagrangian of each optimization problem using the Karush-Kuhn-Tucker (KKT) approach, generalizing the method of Lagrange multipliers for problems with inequality constraints, as opposed to pure equality constraints.

$$
\begin{aligned}
& \mathbb{L}_{f u e l}(f)=\sum_{j} f_{j} C_{j}+\sum_{j}\left(f_{j}-S_{j}\right) \mu_{j}+\left(F-\sum_{j} f_{j}\right) \pi-\sum_{j} f_{j} \phi_{j} \\
& \mathbb{L}_{\text {power }}\left(x_{i}\right)=F p+\lambda\left(D-\sum_{i} x_{i}\right)+\beta\left(F-\sum_{i} x_{i} H_{i}\right)-\sum_{i} x_{i} \chi_{i}-F \zeta
\end{aligned}
$$

For problems (1) and (2) the Lagrangian is represented by $\mathbb{L}_{f u e l}\left(f_{j}\right)$ and $\mathbb{L}_{\text {power }}\left(x_{i}, p\right)$ in equations (A1) and (A2), respectively. We introduce the following orthogonal complementarity variables, or Lagrange multipliers, for each constraint in the original primal problems. Variables $\mu, \pi, \lambda$, and $\beta$ are the non-negative dual variables on (1.1), (1.2), (2.1), and (2.2), respectively. We also introduce $\phi_{j^{\prime}} \chi_{i^{\prime}}, \zeta$ as the duals on the non-negativity of $f_{j}, x_{i}$ and $F$, respectively.

Notice here that the equilibrium condition for the price of fuel paid by the utility is external to both primal problems. It will be introduced later after deriving the optimality conditions for each sector.

The Lagrangian consists of the objective value (the total cost of each sector) plus terms from the constraints multiplied by their dual variables. When constructing the Lagrangian, the terms in each inequality constraint must reflect the direction of optimization. In a cost minimization, the terms are evaluated on the left-hand side of the smaller than or equal to inequality in order to have the correct sign. The opposite is true when the objective function is being maximized. The terms are multiplied by the corresponding orthogonal dual variables, summed over all indices and added to the scalar objective value.

Next, we impose the condition that the gradient of the Lagrangian with respect to its independent variables equals zero in the optimal solution, as it should be a stationarity point.

$$
\begin{array}{ll}
\frac{\partial \mathbb{L}_{\text {fuel }}}{\partial f_{j}}=C_{j}+\mu_{j}-\pi-\phi_{j}=0 & \\
\frac{\partial \mathbb{L}_{\text {power }}}{\partial x_{i}}=-\lambda-\beta-\chi_{i}=0 & \\
\frac{\partial \mathbb{L}_{\text {power }}}{\partial F}=p+\beta-\zeta=0 & \\
& \\
C_{j}+\mu_{j}-\pi \leq 0 & \perp f_{j} \geq 0 \\
-\lambda-\beta \leq 0 & \perp x_{i} \geq 0 \\
p+\beta \leq 0 & \perp \mathrm{F} \geq 0 \\
f-S \leq 0 & \perp \mu \geq 0 \\
F-f \leq 0 & \perp \pi \geq 0 \\
D-\sum_{i} x_{i} \leq 0 & \perp \lambda \geq 0 \\
F-\sum_{i} x_{i} H_{i} \leq 0 & \perp \beta \geq 0
\end{array}
$$


The stationarity conditions (A3.1), (A3.2), and (A3.3) are converted into complementarity slackness conditions (A4.1), (4.2), and (A4.3), respectively, by substituting the complementary conditions for the non-negativity of the primal variables: $f_{j} \geq 0 \perp \phi_{j} \geq 0, x_{i} \geq 0 \perp \chi_{i} \geq 0$, and $F \geq 0 \perp \zeta \geq 0$. These are complemented by their corresponding dual variables to obtain the system of equations in (A4), also called the KKT conditions. To complete the MCP, we introduce the equilibrium condition for the price of fuel purchased by the utility in equation (A5).

$$
p=\left\{\begin{array}{l}
\pi \\
A
\end{array}\right.
$$

This system of equations holds for marginal cost (competitive) pricing when $p=\pi$ and administered (fixed) pricing when $p=A$. The former defines the linear complementarity problem (LCP), equivalent to standard cost minimization problem combining (1) and (2). We can also modify the MCP to represent average cost pricing or a price cap. The main advantage is the price linkage between sectors (A5) can be defined explicitly, which is not possible in standard economic optimization problems. 
Below is the GAMS code for the two-sector problem introduced in equation blocks (1) and (2), and the four pricing options discussed in the main text: perfect competition, administered (or fixed) prices, average cost, and price cap. Following the equation definitions, we provide the emp.info and model solve statement used to construct the equilibrium problem for each pricing option. The emp.info incorporates the complementarity slackness conditions (5.1) - (5.3) for the price cap rule and the additional quota constraint (5.4) for all non-competitive prices. These equations are labeled as EQ5_1, EQ5_2, EQ5_3 and EQ5_4, respectively. The DualVar option is used to declare sigma as the dual variable on (5.2), and DualEqu to declare (5.1) and (5.3) as the complementarity slackness constraints for $q$ and $r$, respectively.

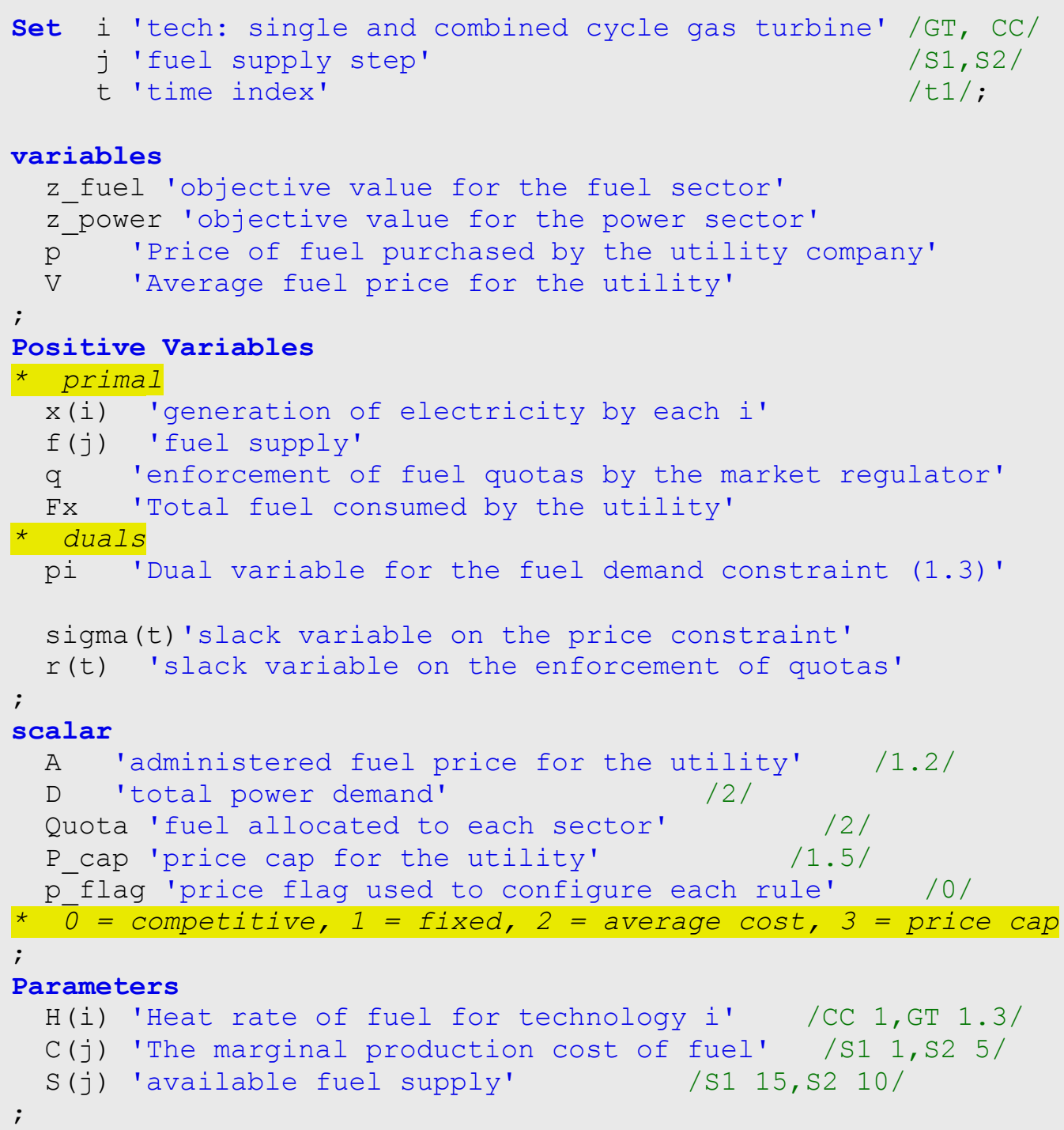




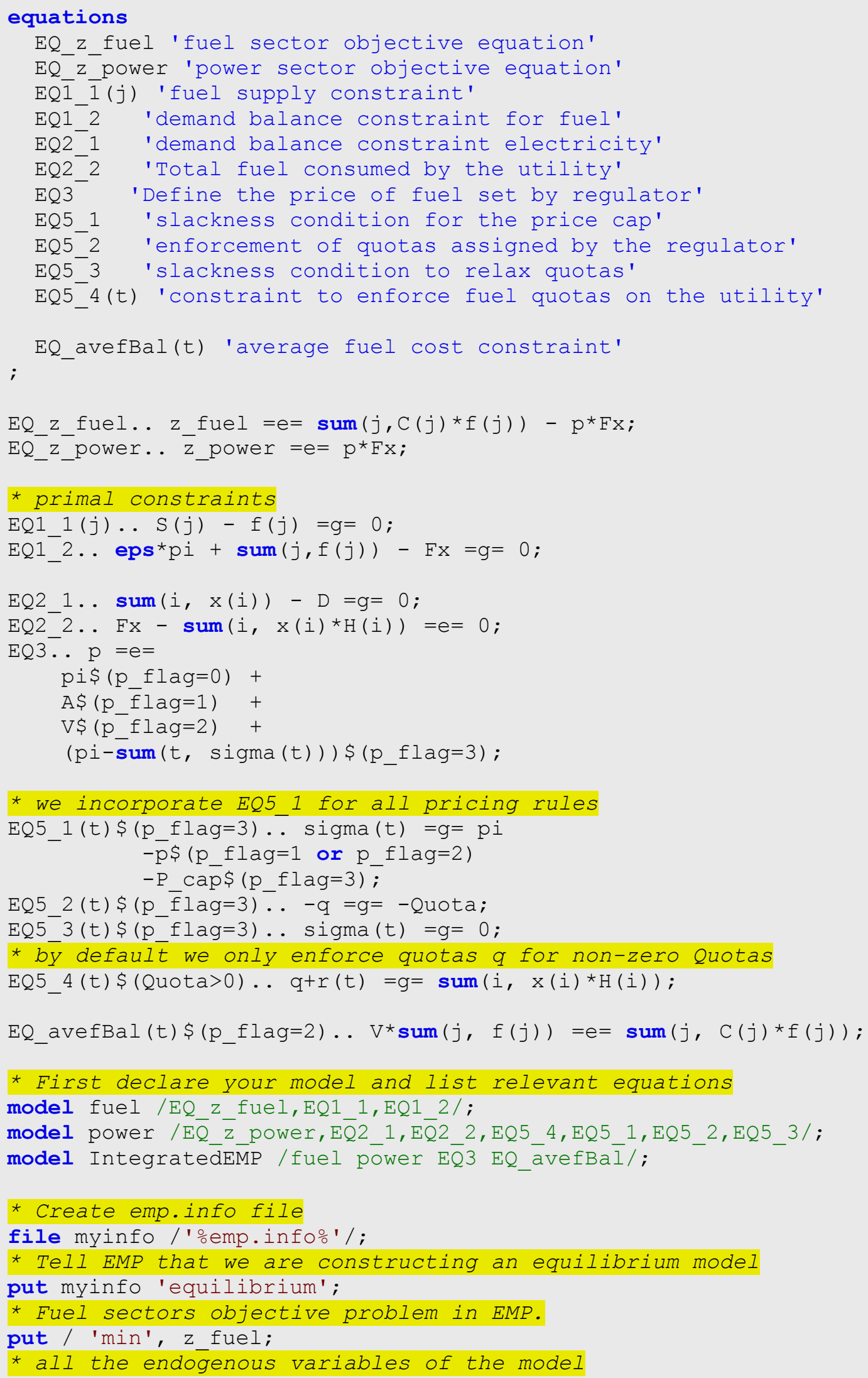




\section{Appendix 2}

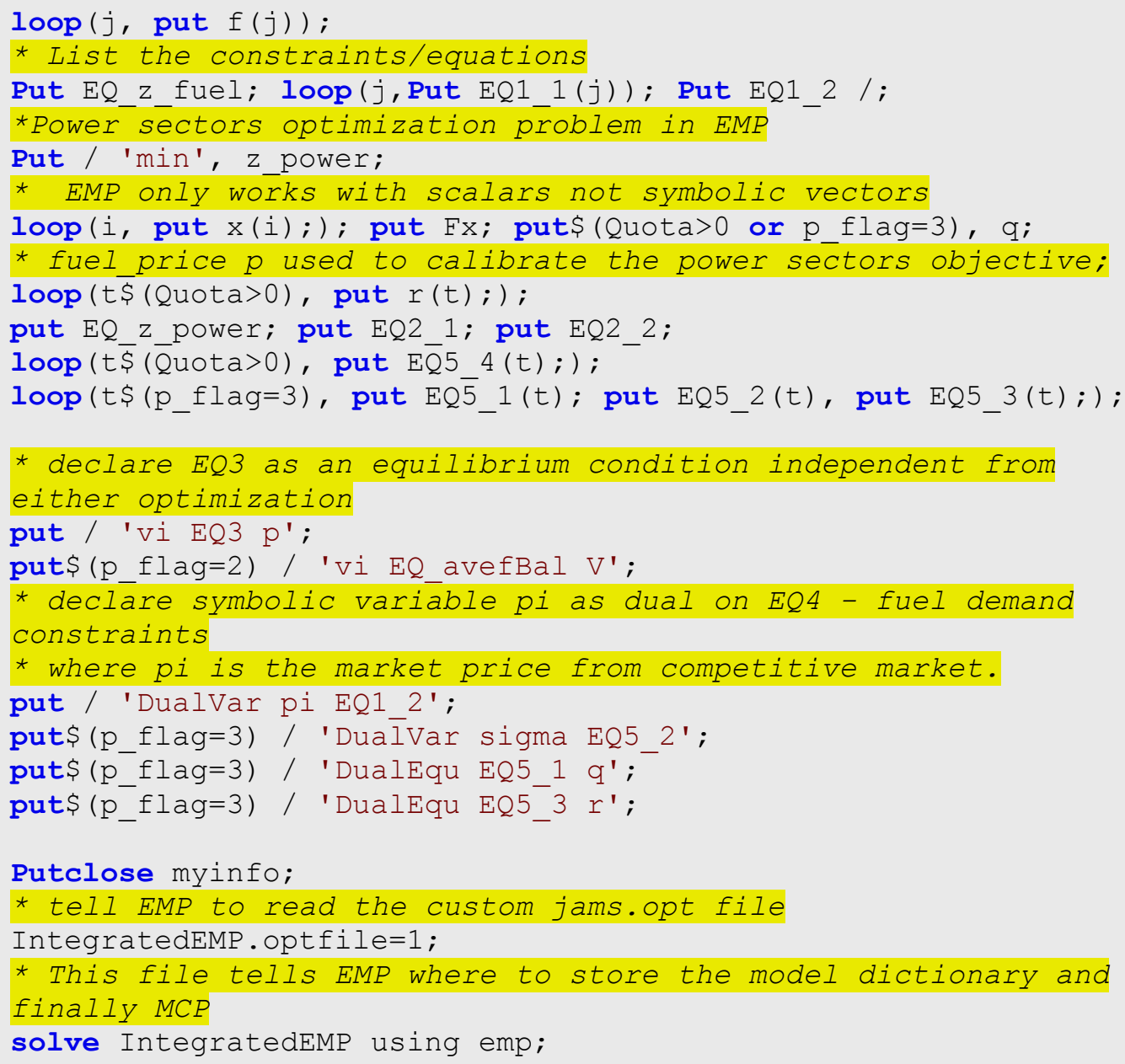




\section{Appendix 3: Building the reliability options model in EMP}

To build the reliability options model described in the main text, we must construct the optimality conditions of the generator's problem. We first derive the MCP manually and then demonstrate how to build it in GAMS using EMP.

$$
\begin{aligned}
& \mathbb{L}_{j}=\sum_{l} r_{j l} \cdot o_{l}+\sum_{i k l}\left(p_{k l}-C_{i j k}\right) x_{j k l} \rho_{k}-\sum_{k l} s_{k l}^{+} r_{j l} \rho_{k}+\sum_{k l}\left(\sum_{i j^{\prime}} x_{i j^{\prime} k l}-q_{k l}\right) \rho_{k} \alpha_{j k l}+ \\
& \sum_{i k l}\left(E_{i j}-x_{i j k l}\right) \lambda_{i j k l} \rho_{k}+\sum_{l}\left(\sum_{i} E_{i j}-r_{j l}\right) \gamma_{j l}+\sum_{i j k l} x_{i j k} \delta_{i j k l} \rho_{k}+\sum_{j l} r_{j l} \varepsilon_{j l}
\end{aligned}
$$

First let $\mathbb{L}_{j}$ represent the generator's Lagrangian as constructed from the generator problem (8). In equation (A6) we construct the Lagrangian by defining the orthogonal complementarity variables, $\alpha_{k l^{\prime}}$ $\lambda_{i j k l}$ and $\gamma_{k l}$ for constraints (8.1), (8.2) and (8.3), respectively. We also introduce $\delta_{i j k l}$ and $\varepsilon_{j l}$ as the duals corresponding to the non-negativity of $x_{i j k}$ and $r_{j l}$, respectively.

$$
\begin{array}{ll}
p_{k l} \geq \lambda_{i j k l}+C_{i j k}+\left(1-X_{j}\right) b_{k l} x_{j k l}-\alpha_{j k l} & \perp x_{i j k l} \geq 0 \\
\gamma_{j l} \geq 0 & \perp r_{j l} \geq 0 \\
\sum_{i j} x_{i j k l} \geq q_{k l} & \perp \alpha_{j k l} \geq 0 \\
E_{i j} \geq x_{i j k l} & \perp \lambda_{i j k l} \geq 0 \\
\sum_{i} E_{i j} \geq r_{j l} & \perp \gamma_{j l} \geq 0 \\
p_{k l}=a_{k l}-b_{k l} \sum_{i j} x_{i j k l} & \\
s_{k l}^{+} \geq p_{k l}-\hat{p} & \perp q_{k l} \geq 0 \\
\sum_{j} r_{j l} \geq q_{k l} & \perp s_{k l}^{+} \geq 0
\end{array}
$$

Next we formulate the optimality conditions (A7.1) and (A7.2) by taking the partial derivatives of $\mathbb{L}_{j}$ against the primary variables $x_{i j k}$ and $r_{j l}$, respectively, setting them to zero in equilibrium and substituting in the non-negativity conditions. In (A7.1) we introduce the conjectural variation $X_{j}=\partial \sum_{j^{\prime} \neq j} x_{i j^{\prime} k l} / \partial x_{i j k l}$ when taking the partial derivative of the energy price with respect to the generator's production. This represents how generator $j$ will adjust its own production in response to a change in the aggregate production of all other generators, $j^{\prime} \neq j$. We assume the conjectural variation is the same across all technologies, scenarios and load segments. Under the assumption that a generator behaves competitively, $X_{j}=-1$, whereas in an oligopolistic market it is assumed that the generator does not change its production in response to the other competitors (i.e., $X_{j}=0$ ). Under competitive behavior the corresponding market power term in (7.1) drops out, and generators act as price takers. In an oligopoly, the market power term $b_{k l} x_{j k l}$ is added to the optimality condition such that generators observe the impact of their production on prices. As a result, they will withhold capacity in order to maximize their marginal revenue. 
Notice the negative $\alpha_{j k l}$ term in (A7.1). This represents the marginal value of all the generator's commitments $\left(\sum_{i j} x_{i j k l}\right)$ to honor the pool of reliability options $q_{k l}$ that can be exercised by the buyer. If generators sell options ahead of the energy market, they are required to supply the corresponding capacity, reducing their ability to exercise market power by withholding supplies. The dual $\alpha_{j k l}$ represents the incentive given to generators to sell reliability options in advance, counteracting the exercise of market power in the real-time energy market.

The complete KKT conditions of the wholesale electricity market with reliability options in (A7) are constructed by adding the original primal constraints of (8) and the market clearing conditions from (6) and (7).

In the following GAMS code, we demonstrate how to construct (A7) using EMP as a Cournot-Nash equilibrium model with oligopolistic generators. See the note below the \$MACRO price $(\mathrm{k}, \mathrm{l})$ below the list of equation declarations for an explanation of how to switch between oligopolistic and competitive representations of firm behavior.

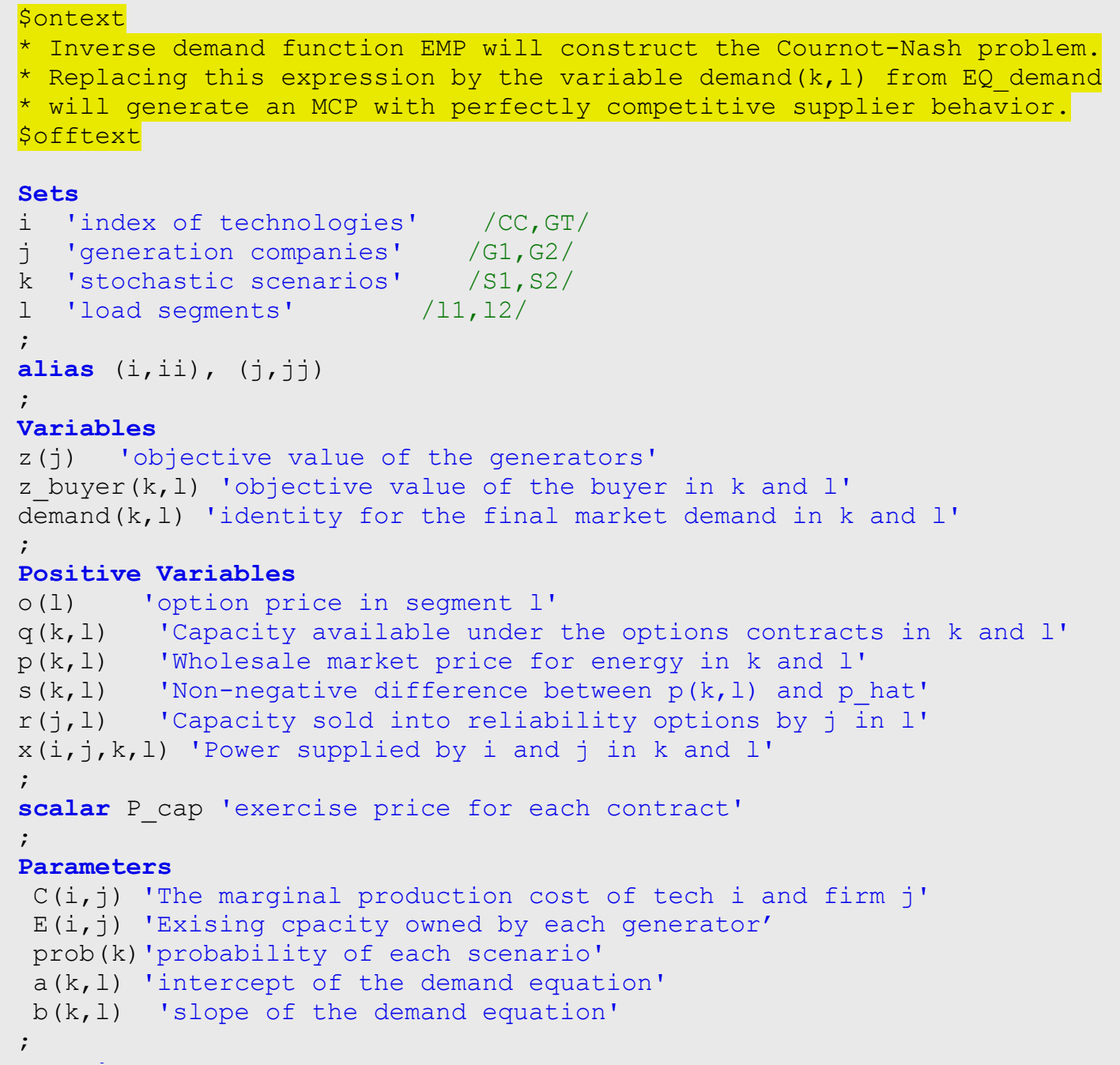




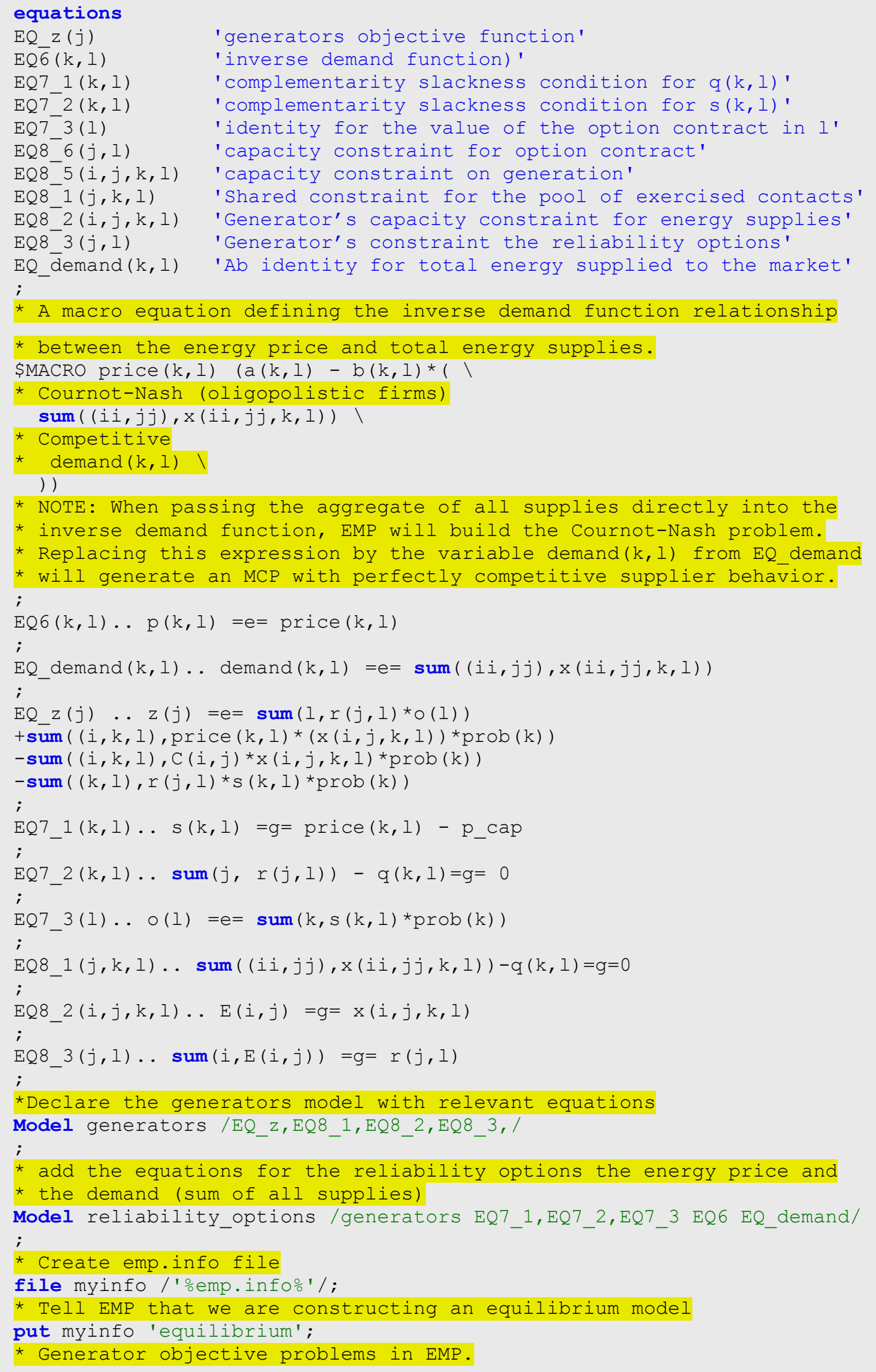




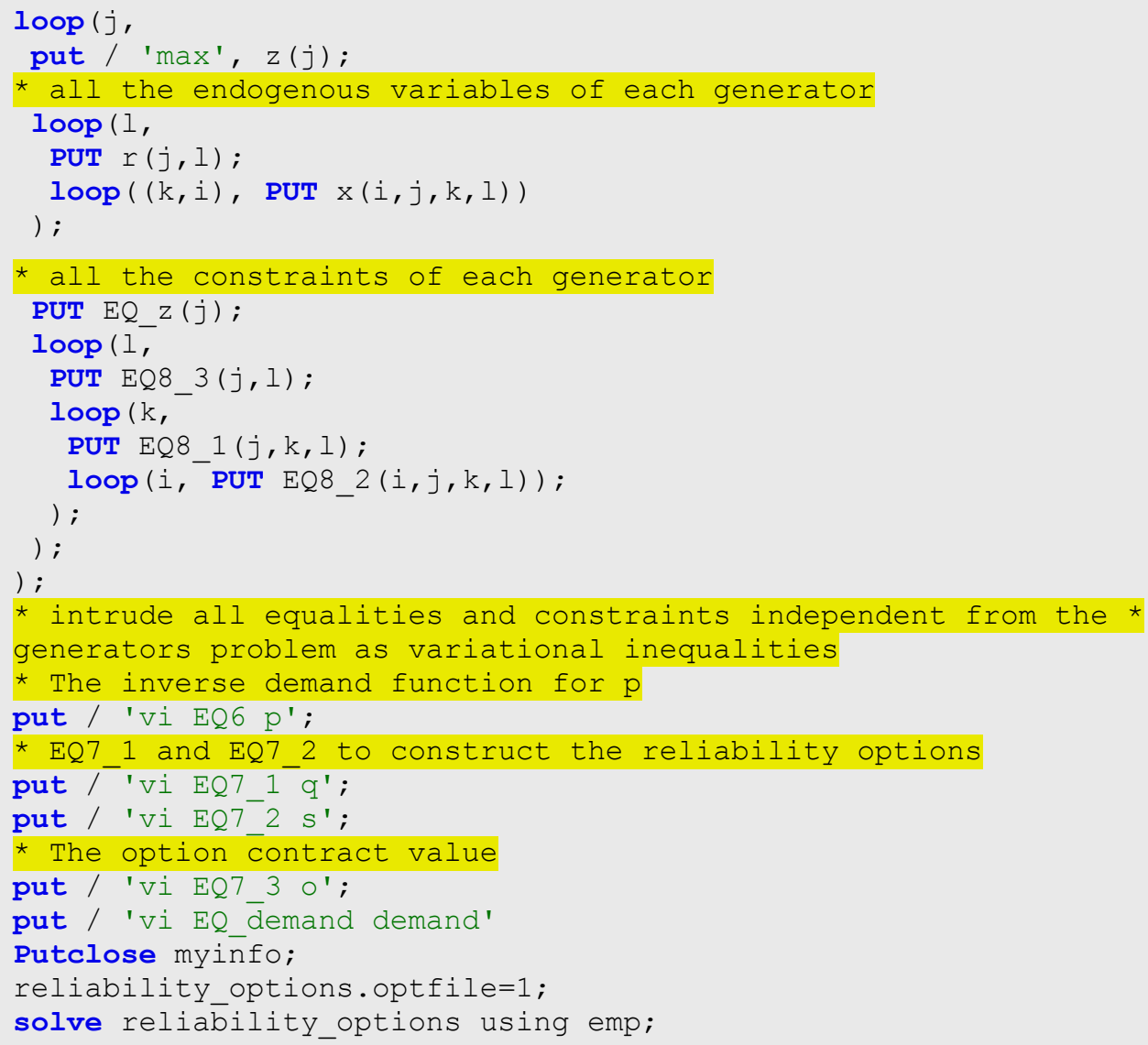




\section{About the Authors}

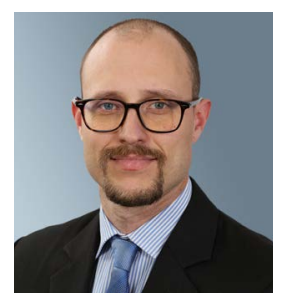

\section{Bertrand Rioux}

Bertrand is a research fellow specializing in energy systems modeling. $\mathrm{He}$ holds an M.S. degree from KAUST with a specialization in computational fluid dynamics.

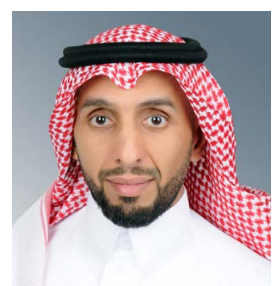

\section{Abdullah Al Jarboua}

Abdullah is a senior research analyst specializing in energy systems and energy economics modeling. He holds an M.S. degree in Computer Science from KAUST.

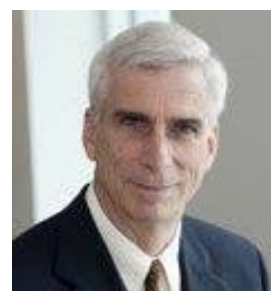

\section{Frederic Murphy}

Frederic is a senior visiting fellow and professor emeritus, Temple University. He has a Ph.D. in Operations Research and a B.A. in Mathematics from Yale University.

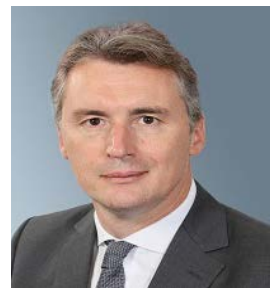

\section{Axel Pierru}

Axel is a senior research fellow and program director at KAPSARC. He has a Ph.D. in Economics from Pantheon-Sorbonne University.

\section{About the Project}

The KAPSARC Energy Model (KEM) project first started in 2013. It has since evolved into a general modeling toolkit used to study price regulation and market reform in large energy economies. Starting in 2018, the Extended Mathematical Programming (EMP) framework was incorporated into KEM to help streamline the development and management of existing and new applications. 
INAPSARC

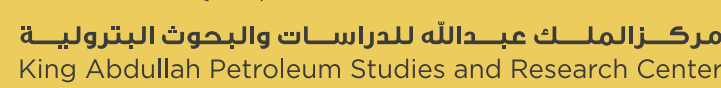

www.kapsarc.org 